technique for monitoring viral infection in live mice.

Mouse models of herpes simplex virus type 1 (HSV-1), which infects an estimated $80 \%$ of adults worldwide, have allowed scientists to glean valuable information on viral infection, progression, and treatment. Nevertheless, current techniques require the sacrifice of mice at various time points, precluding the real-time serial study of viral infection in the same animal.

David A. Leib and colleagues used a recombinant HSV-1 engineered to carry the gene that produces luciferase, the enzyme that produces bioluminescence when exposed to its substrate, luciferin (J. Virol., December 2002). Leib's team inoculated mice by injecting the recombinant virus into their abdomen, brain, cornea, or footpad. Then, every day for nine days, the team injected the mice with luciferin. They then anesthetized the mice and used a camera that detects the light emitted by luciferase to monitor infection in the mice. The image produced by the camera showed the location and amount of virus in a mouse as areas of color. The team found the viral titers correlated with bioluminescence, and they were able to use this technique to evaluate the efficacy of treatment with the anti-herpes drug valacyclovir.

"This methodology will be useful for looking in real time [at] how vaccines and antivirals can alter the spread and tropism of viral infection [and can be used for] predictive studies of the consequences of spread, since we can visualize the spread without sacrifice of the animal," according to Leib.

For the next step in this research, Leib tells Lab Animal, "We will be using this methodology to examine the role of the immune response in shaping the outcome of infection."

\section{Drug Addiction in Teenage Mice}

As if mimicking a human reaction, adolescent mice are more sensitive to addictive drugs than either infant or adult mice, according to the results of a recent study published in the Journal of Neuroscience (1 November 2002).

Although illicit drug use sharply increases during human adolescence, there is little information about the molecular mechanisms that may underlie this phenomenon. Now, Michelle E. Ehrlich of the Nathan Kline Institute (Orangeburg, NY) and New York University School of Medicine (New York, NY) and colleagues report the results of giving post-weanling (24-day-old), periadolescent (33-day-old), and adult (60-day-old) mice intraperitoneal injections of cocaine or amphetamine daily for seven days. The team measured protein levels in the mouse brains and found that chronic exposure to these psychostimulants was associated with a greater increase in levels of the transcription factor $\Delta F o s B$ in the brains of periadolescent mice, compared to post-weanlings or adults. These increases occurred specifically in the brain regions associated with motor activity and the reward system. This is the first study to compare neurochemical responses of animals to psycho-stimulants at different ages. The results may help explain the unique responses of the adolescent brain to psychostimulants.

$-T . S$.

\section{Errata}

- In the article, "Matching Protocols to Grant Proposals," appearing in the October 2002 issue, the Office of Laboratory Animal Welfare (OLAW) was inadvertently misquoted regarding its position on the institutional requirement that protocols and grant proposals be congruent. Please see http://www.labanimal.com/col/ prot0799.htm to read the correct quote.

- In the article, "Email Lists in Laboratory Animal Science," appearing in the November 2002 issue, the email address for the CompMed list was incorrect. The correct address is listserv@listserv.aalas.org. 